\title{
Topographic Organization of the 'Third-Tier' Dorsomedial Visual Cortex in the Macaque
}

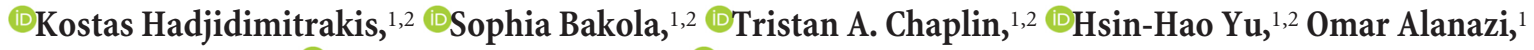 \\ Jonathan M. Chan, ${ }^{1}{ }^{\odot K a t r i n a ~ H . ~ W o r t h y, ~}{ }^{1}$ and $\odot$ Marcello G.P. Rosa ${ }^{1,2,3}$ \\ ${ }^{1}$ Department of Physiology, Biomedicine Discovery Institute, Monash University, Clayton, Victoria 3800, Australia, ${ }^{2}$ Australian Research Council Centre of \\ Excellence for Integrative Brain Function, Monash University Node, Monash University, Clayton, Victoria 3800, Australia, and ${ }^{3}$ School of Biomedical \\ Sciences (previously Vision, Touch and Hearing Research Centre, Department of Physiology and Pharmacology), The University of Queensland, Brisbane \\ QLD 4072, Australia
}

The boundaries of the visual areas located anterior to $\mathrm{V} 2$ in the dorsomedial region of the macaque cortex remain contentious. This region is usually conceptualized as including two functional subdivisions: the dorsal component of area V3 (V3d) laterally and another area named the parietooccipital area (P0) or V6 medially. However, the nature of the putative border between V3d and P0/V6 has remained undefined. We recorded the receptive fields of multiunit clusters in male macaques and reconstructed the locations of recording sites using histological sections and computer-generated maps. Immediately adjacent to dorsomedial V2, we observed a representation of the lower contralateral quadrant that represented the vertical meridian at its rostral border. This region formed a simple eccentricity gradient from $\sim<5^{\circ}$ in the annectant gyrus to $>60^{\circ}$ in the parietooccipital medial sulcus. There was no topographic reversal where one would expect to find the border between $\mathrm{V} 3 \mathrm{~d}$ and P0/V6. Rather, near the midline, this lower quadrant map continued directly into a representation of the peripheral upper visual field without an intervening lower quadrant representation. Therefore, cortex previously assigned to the medial part of $\mathrm{V} 3 \mathrm{~d}$ and to $\mathrm{P0} / \mathrm{V} 6$ forms a single map that includes parts of both quadrants. Together with previous observations that V3d and PO/V6 are densely myelinated relative to adjacent cortex and share similar input from V1, these results suggest that they are parts of a single area (for which we suggest the designation V6), which is distinct from the one forming the ventral component of the third-tier complex.

Key words: dorsal stream; parietooccipital sulcus; primate; receptive fields; visual cortex; visuotopic organization

\section{Significance Statement}

The primate visual cortex has a large number of areas. Knowing the extent of each visual area and how they can be distinguished from each other is essential for the interpretation of experiments aimed at understanding visual processing. Currently, there are conflicting models of the organization of the dorsomedial visual cortex rostral to area V2 (one of the earliest stages of cortical processing of vision). By conducting large-scale electrophysiological recordings, we found that what were originally thought to be distinct areas in this region (dorsal V3 and the parietooccipital area P0/V6), together form a single map of the visual field. This will help to guide future functional studies and the interpretation of the outcomes of lesions involving the dorsal visual cortex.

\section{Introduction}

Despite four decades of research, there is still controversy regarding the boundaries of visual areas in the "third-tier" cortex; that

Received Jan. 11, 2019; revised April 19, 2019; accepted April 20, 2019.

Author contributions: K.H., S.B., T.A.C., H.-H.Y., K.H.W., and M.G.P.R. performed research; K.H., S.B., T.A.C., H.-H.Y., O.A., J.M.C., and M.G.P.R. analyzed data; K.H. wrote the first draft of the paper; K.H., S.B., T.A.C., H.-H.Y., J.M.C., and M.G.P.R. edited the paper; M.G.P.R. designed research.

This work was supported by the Australian Research Council (Grants DE120102883, DP140101968, CE140100007, and DE120102883), the National Health and Medical Research Council (Grants 1020839 and 1082144), and the European Union (Grant H2020-MSCA-734227-PLATYPUS). S.B. was supported by EU Fellowship FP7-PEOPLE-2011-IOF 300452. T.A.C. was supported by an Australian Postgraduate Award and a Monash University Faculty of Medicine Bridging Postdoctoral Fellowship. We thank Rowan Tweedale for assistance during many phases is, the areas located rostral to the second visual area (V2) (Allman and Kaas, 1975). Here, we focus on the parts of this complex located in the dorsomedial region of the macaque brain, including the medial part of the lunate sulcus, annectant gyrus, parietooccipital sulcus, and mesial surface of the brain (Fig. 1). The

of this project, including participation in experiments and critical reading of earlier versions of the manuscript and Janssen-Cilag for providing the sufentanil citrate used in some of the experiments.

The authors declare no competing financial interests.

Correspondence should be addressed to Kostas Hadjidimitrakis at Konstantinos.Chatzidimitrakis@monash.edu or Marcello G.P. Rosa at Marcello.Rosa@monash.edu.

https://doi.org/10.1523/JNEUROSCI.0085-19.2019

Copyright $\odot 2019$ the authors 

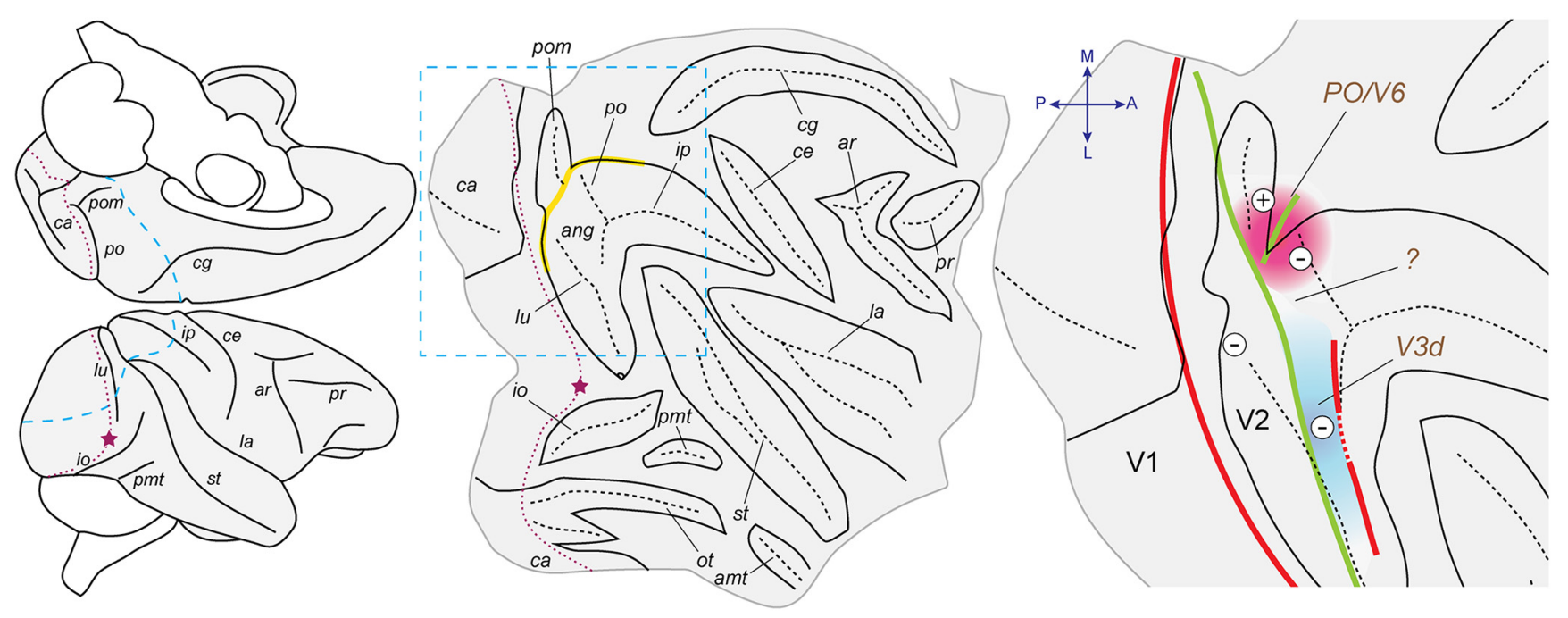

Figure 1. Location of the region of interest (dorsomedial third-tier cortex) and current model of its subdivision into areas. Left, Medial (top) and lateral (bottom) views of the right hemisphere of a macaque brain showing the location of the region explored in the present study (caudal to the blue dashed line). For orientation, the location of the representation of the center of the fovea in the primary visual area (V1) is indicated by a star and the border between V1 and the second visual area (V2) by a dotted red line. Center, Unfolded representation of the macaque brain based on a reconstruction prepared with CARET (Van Essen et al., 2001). In this map, the lips of the sulci are indicated in continuous black line and their main internal folds by black dashed lines. The rectangle shows the region of interest. The yellow highlight indicates the approximate transition between cortex located in the banks of the lunate, parietooccipital, and intraparietal sulci and cortex exposed in the midline, including the parietooccipital medial sulcus (this landmark is also used in Fig. 4 to show the relative location of recording sites in each case). Right, Diagrammatic representation of the model that we tested in this study (Ungerleider and Desimone, 1986; Colby et al., 1988; Gattass et al., 1988; Gamberini et al., 2015), according to which the cortex rostral to dorsomedial area V2 contains two representations of the lower visual field, one within the dorsal subdivision of area V3 (V3d, blue) and another in the parietooccipital area (P0, or V6, pink). The question mark indicates the possibility that another area, PIP (Felleman and Van Essen, 1991) may separate V3d from P0/V6. In this diagram, thick red lines indicate representations of the vertical meridian of the visual field, thick green lines indicate representations of the horizontal meridian, minus signs indicate representations of the lower contralateral quadrant, and plus signs indicate a representation of the upper contralateral quadrant. $A, P, M$, and $L$ indicate anterior, posterior, medial, and lateral, respectively. amt, anterior middle temporal sulcus; ang, annectant gyrus; ar, arcuate sulcus; ca, calcarine sulcus; ce, central sulcus; cg, cingulate sulcus; io, inferior occipital sulcus; i.p., intraparietal sulcus; la, lateral sulcus; lu, lunate sulcus; ot, occipitotemporal sulcus; pmt, posterior middle temporal sulcus; p.o., parietooccipital sulcus; pom, parietooccipital medial sulcus; pr, principal sulcus; st, superior temporal sulcus.

traditional view has been that there are at least two subdivisions in this region. Laterally, in the lunate sulcus and annectant gyrus, most studies indicate the existence of a dorsal component of area V3 (V3d) ( Gattass et al., 1988). Medially, the cortex along the banks of the parietooccipital sulcus and mesial surface is usually assigned to a different region, termed the parietooccipital area (PO) (Colby et al., 1988) or V6 (Galletti et al., 1999) (Fig. 1). Although proposals regarding the boundaries of $\mathrm{PO} / \mathrm{V} 6$ continue to evolve (Gamberini et al., 2015), it is generally agreed that receptive fields of neurons in this region emphasize peripheral vision. Moreover, models proposed by various groups converge on the idea that PO/V6 encompasses a representation of the lower quadrant in the parietooccipital sulcus, as well as a representation of the upper visual field on the mesial surface of the hemisphere. Models proposed for the organization of PO/V6 in capuchin monkeys (Neuenschwander et al., 1994) and humans (Pitzalis et al., 2006) reflect these features.

Visual areas corresponding to early stages of visual processing each form a representation of the visual field (Van Essen and Zeki, 1978; Sereno et al., 1995; Rosa et al., 1997). Therefore, if V3d and $\mathrm{PO} / \mathrm{V} 6$ are distinct areas, then it would be expected that they form separable visuotopic maps. In particular, as depicted in most studies, there should be distinct representations of the peripheral lower quadrant near their common boundary (Fig. 1). However, the transition between V3d and PO/V6 has never been documented in detail, leaving significant room for other interpretations. For example, based on analyses of cortical architectonics, Lewis and Van Essen (2000) indicated that V3d and $\mathrm{PO} / \mathrm{V} 6$ may not be adjacent, being separated by a posterior intraparietal area (PIP). Conversely, based on meta-analysis of pub- lished data, Angelucci and Rosa (2015) proposed that V3d and $\mathrm{PO} / \mathrm{V} 6$ could actually be parts of the same area. Indeed, V3d and $\mathrm{PO} / \mathrm{V} 6$ share significant similarities, such as dense myelination, a projection from the primary visual cortex (V1) that originates in layer $4 \mathrm{~b}$, and large numbers of direction-selective neurons (Felleman and Van Essen, 1987; Felleman et al., 1997; Galletti et al., 2001; Rosa et al., 2009; Pitzalis et al., 2010). They are also reported to have complementary emphases in representation of central (V3d) versus peripheral (PO/V6) visual field. Given the above, it is important to explore the visuotopic organization of the cortex around their putative border to ascertain whether the two-area scheme outlined above does in fact provide the best description of the organization of the dorsomedial third-tier cortex. Bringing clarity to the topographic organization of this region of the macaque cortex is also relevant to understand similarities and differences with New World monkeys, in which a single dorsomedial area (DM) has been proposed to occupy the corresponding region (Rosa and Schmid, 1995; Rosa et al., 2005).

Based on electrophysiological recordings, we found that the cortex anterior to $\mathrm{V} 2$ formed a relatively simple pattern of representation of the lower quadrant, with lateral to medial gradients of increasing eccentricity and receptive field size. However, we also found that this representation merged directly into a representation of the upper quadrant near the midline, thereby forming a single representation of the peripheral visual field. Complementing the results of a recent study of the lateral part of the third-tier complex (Zhu and Vanduffel, 2019), these findings point to a reinterpretation of the areas rostral to dorsal V2 in the macaque. 


\section{Materials and Methods}

Experimental design and statistical analysis. We report on the results of a descriptive study of visuotopic organization of a region of the cerebral cortex based on data obtained in four young adult male macaque monkeys (Macaca fascicularis). The study was designed to collect data in the form of extents of receptive fields of small clusters of neurons (recorded using penetrating electrodes) and the locations of these clusters in the brain (reconstructed in histological sections). Each animal yielded an independent replicate and the conclusions are based on observation of shared features across the four cases. We only used statistical tests to characterize the relationship between receptive field size and eccentricity (see Fig. 10). For this purpose, best-fitting power functions were calculated using a model II (principal axis) regression using data attributed to a cortical area based on location of the recording sites in the cortex relative to visuotopy; receptive field size itself was not used as a criterion for parsing the data into areas. A permutation test was used for comparison between areas.

Preparation. The experimental protocols were approved by the Animal Experimentation Ethics Committees of the University of Queensland and Monash University, which also monitored the welfare of the animals. All procedures complied with the guidelines of the Australian Code of Practice for the Care and Use of Animals for Scientific Purposes. The animals were premedicated with intramuscular injections of diazepam (3 $\mathrm{mg} / \mathrm{kg})$ and atropine $(0.2 \mathrm{mg} / \mathrm{kg})$ and, after $30 \mathrm{~min}$, were anesthetized with ketamine $(50 \mathrm{mg} / \mathrm{kg}$ ) and xylazine $(3 \mathrm{mg} / \mathrm{kg}$; cases $1-3)$, or with a ketamine/Dormitor/butorphanol mixture (0.1 mg/kg, i.m.; case 4). Anesthesia was maintained with additional doses of ketamine $(12 \mathrm{mg} / \mathrm{kg}$; cases $1-3)$ or alfaxan $(8 \mathrm{mg} / \mathrm{kg}$; case 4$)$ throughout surgery. The animals were placed in a stereotaxic frame and, before the beginning of recording sessions, were implanted with a bolt for holding the head while allowing an unobstructed field of vision (Bourne and Rosa, 2003). The femoral vein was cannulated and a craniotomy was performed to expose the dorsal aspect of the occipital and parietal lobes. An acrylic well was fitted to the skull surrounding the craniotomy, a durotomy was performed over the area of interest, and the well was filled with silicon oil to avoid dehydration of the exposed cortex. After the surgical procedures were completed, the animals were administered an intravenous infusion of pancuronium bromide $(0.5 \mathrm{mg} / \mathrm{kg}$, followed by $0.1 \mathrm{mg} / \mathrm{kg} / \mathrm{h})$, combined with sufentanil citrate $(6-8 \mu \mathrm{g} / \mathrm{kg} / \mathrm{h})$, in a solution of sodium chloride $(0.18 \%) / g l u c o s e(4 \%)$ and dexamethasone $(0.4 \mathrm{mg} / \mathrm{kg} / \mathrm{h})$. They were maintained under artificial ventilation with a gaseous mixture of $\mathrm{N}_{2} \mathrm{O} / \mathrm{O}_{2}$ (7:3). Vital signs (electrocardiograph, $\mathrm{PO}_{2}$, and levels of spontaneous activity in the cortex) were continuously monitored and the temperature was maintained between $36.5^{\circ} \mathrm{C}$ and $37^{\circ} \mathrm{C}$ by means of a heating blanket connected to a rectal temperature probe. Mydriasis and cycloplegia were induced by topical applications of atropine $(1 \%)$ and phenylephrine hydrochloride (10\%) eye drops. Appropriate focus was achieved by means of contact lenses with $3 \mathrm{~mm}$ artificial pupils, which brought into focus the surface of a $57.3 \mathrm{~cm}$ radius translucent hemispheric screen centered on the eye contralateral to the cerebral hemisphere to be studied.

Electrophysiology. Tungsten microelectrodes $(\sim 1 \mathrm{M} \Omega)$ guided by stereotaxic micromanipulators (SM-11; Narishige) were inserted in rows along parasagittal planes $\sim 1.5 \mathrm{~mm}$ apart, with direct visualization of the cortical surface used to avoid puncturing major blood vessels. Penetrations extended in most cases to the calcarine sulcus and in many cases to the ventral surface of the cortex, including recordings from portions of $\mathrm{V} 1$ and V2 in these regions. Recording sites were obtained at different depths in each penetration (every 300-500 $\mu \mathrm{m}$ ). Amplification and filtering were achieved via an AM Systems model 1800 microelectrode alternating current amplifier. Transitions between gray and white matter and between gray matter and sulci were determined based on the change in spontaneous activity determined aurally, and the corresponding depths were noted in the protocol to help aid in the histological reconstruction of electrode tracks.

Visual stimuli were monocularly presented under mesopic adaptation levels to the eye contralateral to the cortical hemisphere from which the neuronal recordings were obtained. The eye ipsilateral to the recording hemisphere was occluded. Visual stimulation was achieved by projecting patterns generated by an ophthalmoscope onto a translucent hemispheric screen centered on the nodal point of the contralateral eye, which allowed presentation of patterns up to $90^{\circ}$ eccentricity ( $\mathrm{Yu}$ and Rosa, 2010). At each recording site, receptive fields of single units or small unit clusters were mapped by correlating changes in neural activity with stimulation of specific portions of the visual field. Typical visual stimuli were white spots $\left(1-10^{\circ}\right.$ in diameter) and luminous bars $\left(2-20^{\circ}\right.$ long, $0.2-1^{\circ}$ wide), which moved or flashed $(1 \mathrm{~Hz})$ on the surface of the screen. The stimulus luminance was between 1 and $10 \mathrm{~cd} / \mathrm{m}^{2}$. Receptive fields were drawn as rectangles parallel to the axis of best orientation, or, in the case of large peripheral receptive fields, ovals. The position of each electrode penetration was marked on digital images of the pattern of blood vessels on the cortical surface obtained with a CCD camera. Electrolytic lesions (4-5 $\mu \mathrm{A}$ for $10 \mathrm{~s}$ ) were placed during the experiment to mark the transitions between areas, the end of electrode tracks, and sites of special interest.

Histology. At the end of the experiments, the animals were administered a lethal dose of sodium pentobarbitone ( $100 \mathrm{mg} / \mathrm{kg}$, i.v.), and perfused transcardially with heparinized saline or phosphate buffer, followed by $4 \%$ paraformaldehyde in $0.1 \mathrm{~m}$ phosphate buffer, $\mathrm{pH}$ 7.4. The brains were removed from the skull, blocked, and cryoprotected by immersion in buffered solutions of sucrose (10-30\%). Once the brains sank in the $30 \%$ sucrose solution, parasagittal sections (40 or $50 \mu \mathrm{m}$ ) were obtained using a cryostat. Adjacent series were stained for Nissl substance, cytochrome oxidase, and myelin using the Gallyas (1979) procedure (Fig. 2). All sections were coverslipped with dibutylphthalate polystyrene xylene (DPX) after dehydration in ethanol and clearing with xylene.

The positions of recording sites were reconstructed in serial sections based on histological observation of gliosis caused by the electrode tracks (Fig. 2), electrolytic lesions, and transitions between the gray and white matter. Shrinkage due to histological processing was estimated by comparing the distances between the electrode tracks in the sections with the microdrive readings.

Data analysis. The horizontal and vertical meridians of the visual field were estimated during the experiment by assuming that the former was aligned with the elevation of the center of the optic disk and that the latter corresponded to a vertical line $\sim 15^{\circ}$ from the center of this retinal landmark. These estimates were refined during the data analysis using well established features of the visual topography of the macaque cortex as references: namely, we assumed that receptive fields recorded at the V1/V2 histological border overlapped with the vertical meridian of the visual field and that receptive fields recorded from neurons near the anterior border of V2 represented the horizontal meridian (Van Essen and Zeki, 1978; Gattass et al., 1981). Repeated plotting of small receptive fields in these areas demonstrated that eye drift did not occur under the dose of pancuronium bromide that we used.

Receptive fields were digitized as rectangles on the spherical surface and all subsequent calculations used appropriate geodesic corrections (Yu and Rosa, 2010). In the case of some of the large peripheral receptive fields, the oval shapes were approximated by a rectangle centered on the same position to facilitate the calculations. The centers of the receptive fields were calculated as the mean values of the azimuth and elevation within the sectors of the visual field that they encompassed, their eccentricities as the geodesic distance between the centers of the receptive field and the intersection between the vertical and horizontal meridians, the polar angle as the angle between the centers of the receptive fields and the horizontal meridian, and the receptive field sizes as the square root of the surface areas of the rectangles that represented them (Yu and Rosa, 2010).

Three-dimensional and 2D reconstructions of the cortical surface were generated with the software CARET (Van Essen et al., 2001). Sections were scanned and aligned with graphics software (Adobe Illustrator) using pinholes created before sectioning as a reference. Layer 4 contours from each case were traced manually on the digitized sections and registered. The contours were then imported into CARET to reconstruct the 3D surface models based on a triangular mesh and irregularities in the mesh introduced due to slight misalignments during mounting 
A

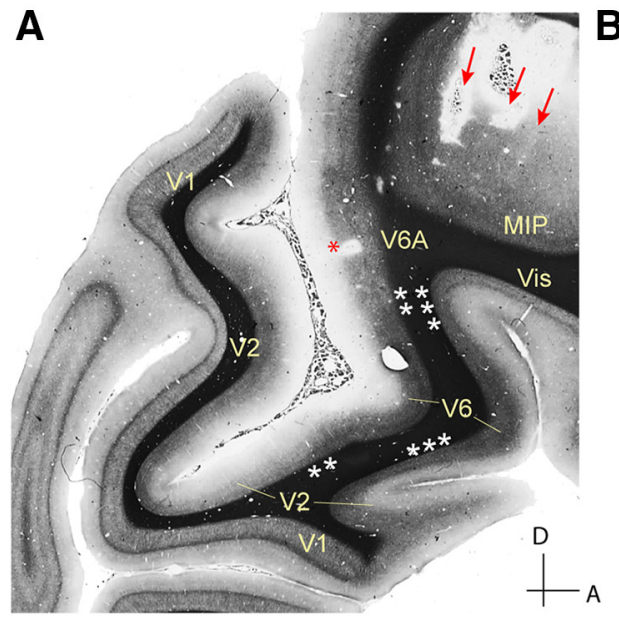

B

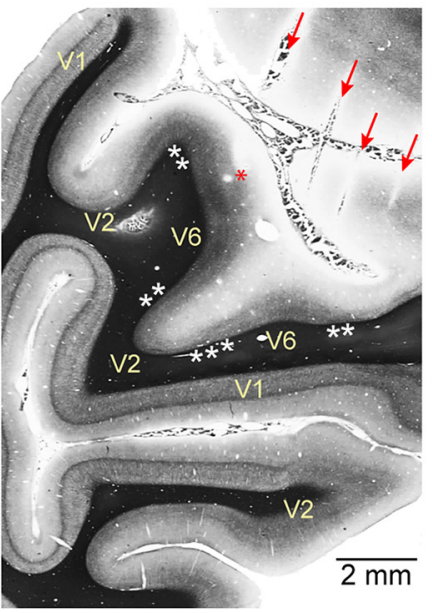

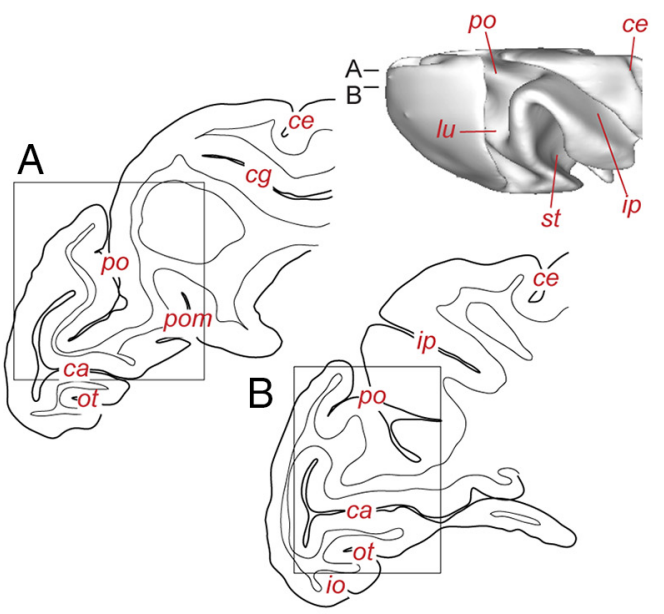

Figure 2. $\quad A, B$, Parasagittal sections stained with the Gallyas (1979) method showing the histological reconstruction of the electrode penetrations and myeloarchitectural characteristics of V2, the dorsomedial third-tier area (V6 in the present nomenclature), and cortex rostral to V6. Both sections are from case 2. The magnified regions are indicated by rectangles in tracings of the sections (right), and their levels are indicated in a dorsal view of the brain reconstructed in CARET. Several electrode tracks (red arrows) and electrolytic lesions (red asterisks) are visible, demonstrating the angle of approach of the electrodes. Rows of white asterisks underlying layer 6 indicate myeloarchitectural transition zones. The V6 cortex appears more myelinated than all adjacent areas, whereas V2 alternates regions of medium and light myelination (Rosa et al., 1988). Other areas are labeled according to Bakola et al. (2017) and Passarelli et al. (2018). MIP, Medial intraparietal area; V6A, visual area $6 \mathrm{~A}$; Vis, visual cortex in the ventral part of the precuneus and rostral part of the parietooccipital medial sulcus). Orientation key (in A): $A$, Anterior; D, dorsal. For abbreviations of the names of sulci, see the legend of Figure 1.
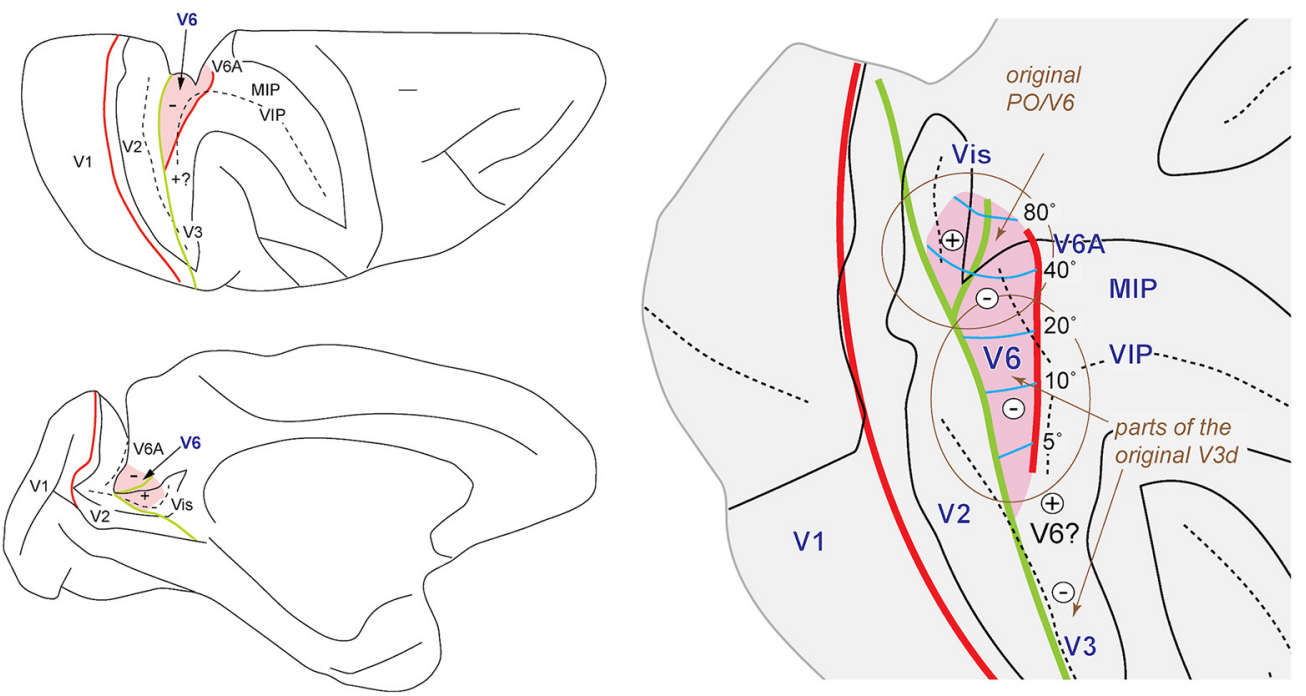

Figure 3. Summary view of the organization of dorsomedial third-tier cortex according to present results. Left, Schematic view of the macaque brain with the sulci partially opened showing the location of the redefined dorsomedial third-tier area V6 (pink) in relation to adjacent subdivisions. Areas in medial cortex are named according to Bakola et al. (2017) and Passarelli et al. (2018). The minus and plus signs indicate representations of the lower and upper quadrants, respectively, red lines indicate representations of the lower vertical meridian, and green lines indicate representations of the horizontal meridian. The plus sign followed by a question mark indicates a region likely to represent the central part of the upper quadrant (Zhu and Vanduffel, 2019), which was not explored in the present experiments. Right, Unfolded representation of the dorsomedial cortex. Area V6 is shown with the same conventions. Isoeccentricity lines are shown in blue. Ovals in this diagram indicate the relationship of the present proposal to previous delineations of the dorsal part of V3 (V3d) and of area P0/V6. In essence, we found that the most medial part of V3d and area P0/V6 form a single complete representation of the lower quadrant, which we assign to area V6, and that V6 also represents at least part of the upper quadrant. According to the present proposal, the designation V 3 is used to refer to the third-tier area that extends from the fundus and anterior bank of the lunate sulcus all the way to the ventral (tentorial) surface of the cortex (Rosa and Manger, 2005).

of the sections were smoothed using MeshLab (Cignoni et al., 2008). The $3 \mathrm{D}$ models were then used to create unfolded (2D) maps of the cortex using standard features of CARET (Chaplin et al., 2013a). Visuotopic coordinates from each recording site were projected on the reconstructed surfaces. Subsequently, visuotopic maps were created by interpolating the eccentricity and polar angle of each recording site's receptive field across the region of interest. We then used an interpolation procedure to estimate coordinates at all mesh nodes using a distance-weighted smoothing algorithm (Sereno et al., 1994; Chaplin et al., 2013b).

\section{Results}

Our main finding is that the third-tier cortex extending from the annectant gyrus to the mesial surface of the macaque brain forms a single representation of the visual field (Fig. 3). This representation, which coincides with the densely myelinated cortex rostral to V2, occupies the territory usually assigned to areas PO/V6, but extends further laterally to include what is traditionally seen as the medial part of V3d. To avoid the introduction of a new no- 
A

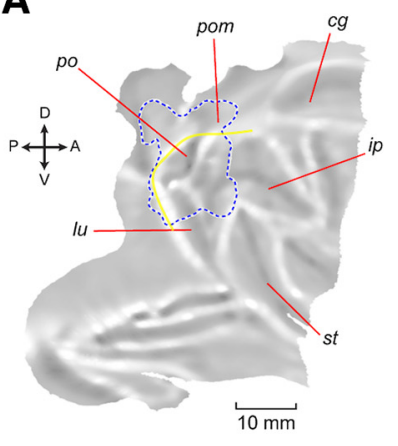

B

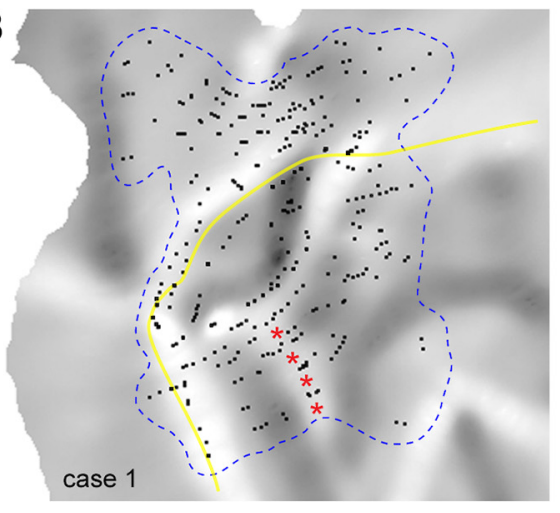

D
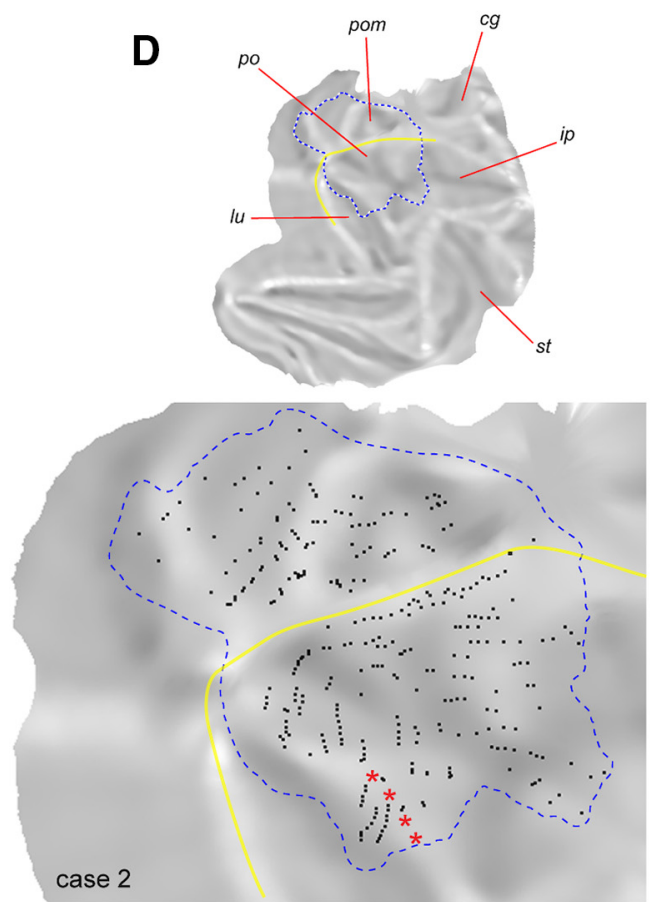

E
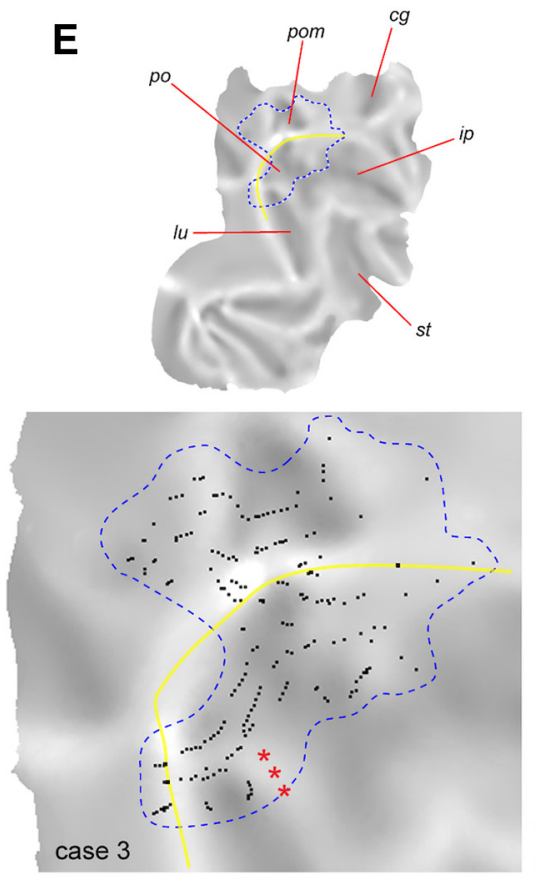

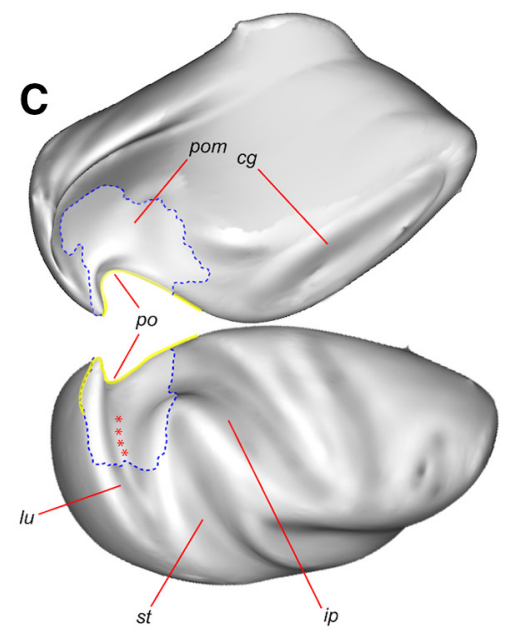

$\mathbf{F}$

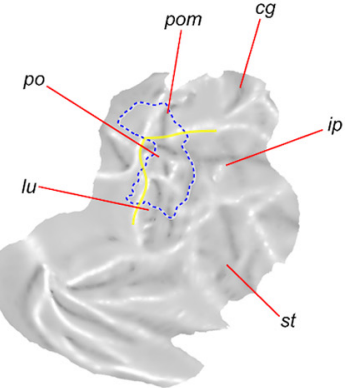

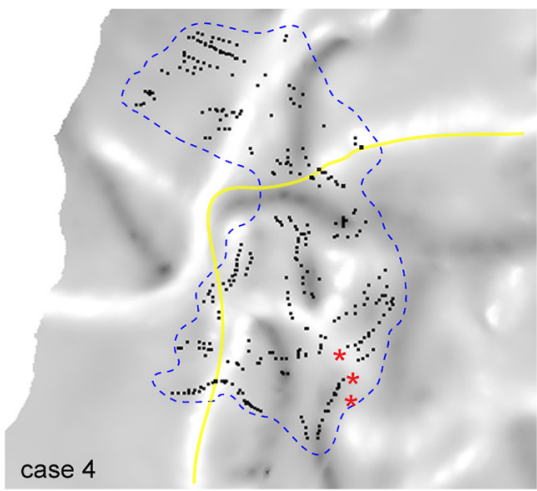

Figure 4. Unfolded reconstructions of the cortex in four cases, showing the locations of recording sites from which visual responses were obtained in dorsal extrastriate areas. $A$, Reconstructed cortex in case 1 showing locations of the sulci (for abbreviations, see Fig. 1). In the insert, A, D, P, and V indicate anterior, dorsal, posterior, and ventral in the brain, respectively. The yellow line indicates the limit between the cortex buried in the lunate-parietooccipital cleft and the cortex exposed in the midline (including the parietooccipital medial sulcus). $\boldsymbol{B}$, Magnified view of the dorsomedial cortex with recording sites indicated as black points. The dashed line indicates the outline of the region explored and used for analysis of visual topography (Fig. 5, bottom). C, Partially inflated view of the brain indicating the location of the region reconstructed in $\boldsymbol{B}$. The medial view (top) was flipped dorsoventrally to facilitate visualization relative to the flat maps. $\boldsymbol{D}-\boldsymbol{F}$, Similar representations of explored region and recording sites in cases 2,3 , and 4 . In all maps, the crown of the annectant gyrus is indicated by the red asterisks.

menclature, here, we have opted to retain the designation V6 for this area based on the fact that, among the three existing name options (V3d, PO, and V6), its boundaries most closely resemble those of V6 as depicted by recent studies (Gamberini et al., 2015). According to this proposal, the designation V3 will be used to refer to an area occupying more lateral and ventral aspects of the third-tier cortex (Fig. 3), which was not covered in our study. We realize that this choice may be controversial given the long history of exploration of this part of the macaque brain, and our views on the relative merits of other options are considered in detail in the Discussion. During the presentation of the Results, we will use the designation "redefined V6" in the following sections to refer to the area that is the focus of the present report.

\section{Recording sites}

The locations of the recording sites that yielded visual receptive fields in the region of interest are illustrated in Figure 4. In each animal, we examined a cortical region extending approximately from the rostral border of V1 to the caudal intraparietal sulcus and from the midline to the medial third of the lunate sulcus. The yellow lines in the unfolded maps (Fig. $4 B, D, E, F$ ) indicate the approximate medial boundary of the region encompassing recording sites that were located in the banks of the lunate, parietooccipital, and intraparietal sulci (see schematic in Fig. 4C). In each case, approximately half of the recording sites were located in the banks of these sulci. For further orientation, red asterisks in Figure 4 also indicate the crown of the annectant 

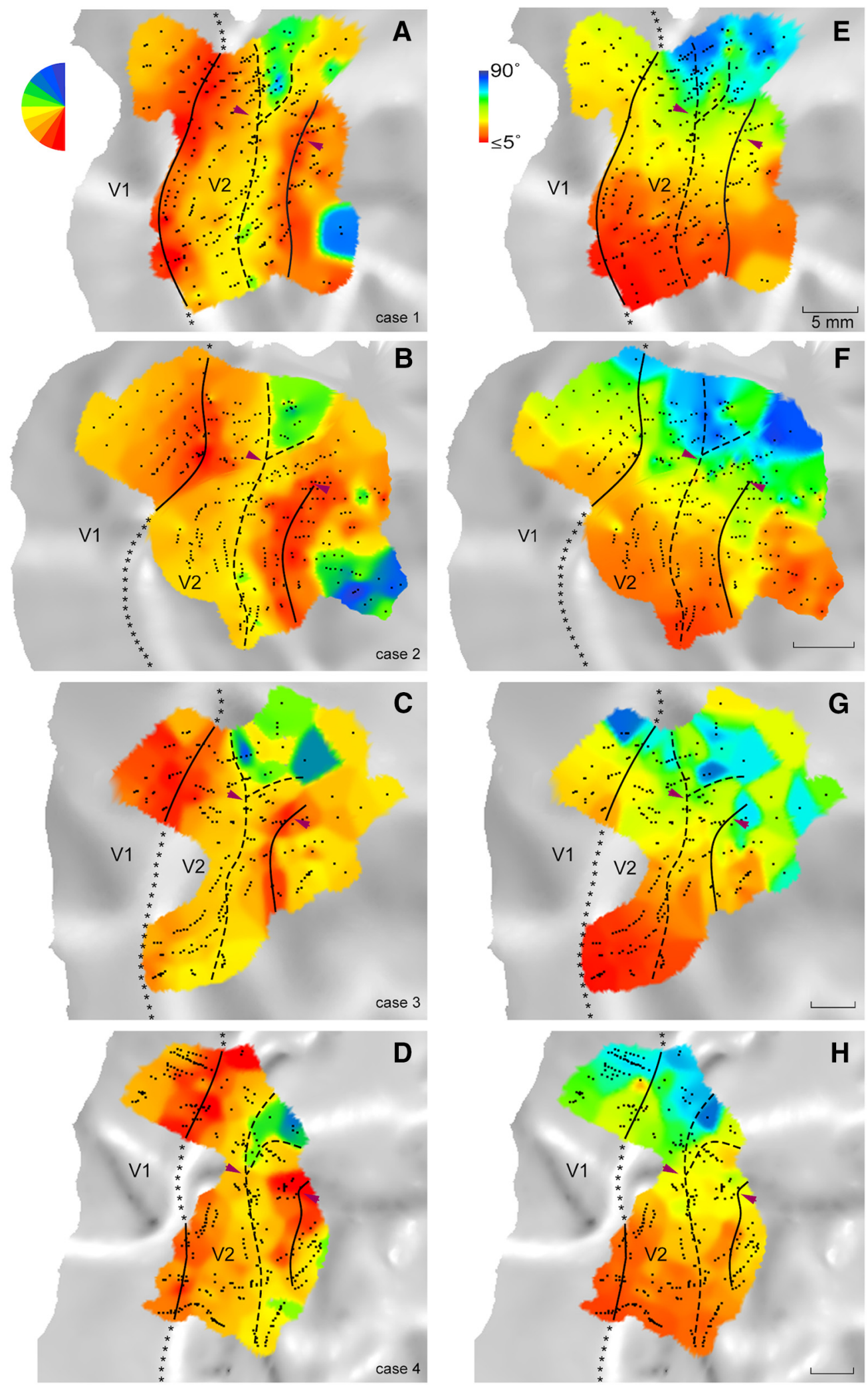

Figure 5. Analysis of visual topography in dorsal extrastriate cortex shown in unfolded representations of the cortex of the four cases. The receptive field polar angle $(\boldsymbol{A}-\boldsymbol{D})$ and eccentricity ( $\boldsymbol{E}-\boldsymbol{H})$ of the recording sites (black points) was interpolated using the method described by Sereno et al. (1994) and colored according to the legends shown adjacent to $\boldsymbol{A}$ and $\boldsymbol{E}$, respectively. In all panels, the black continuous lines were drawn approximately through the middle of the regions of representation of the lower vertical meridian (red tones in $\boldsymbol{A}-\boldsymbol{D}$ ) and the dashed lines through the regions of representation of the horizontal meridian (yellow/light green). The asterisks indicate the location of the V1/V2 cytoarchitectural border in regions where no recordings were obtained. The pairs of arrowheads point to the region near where the parietooccipital sulcus emerges onto the mesial surface (see text for details). 

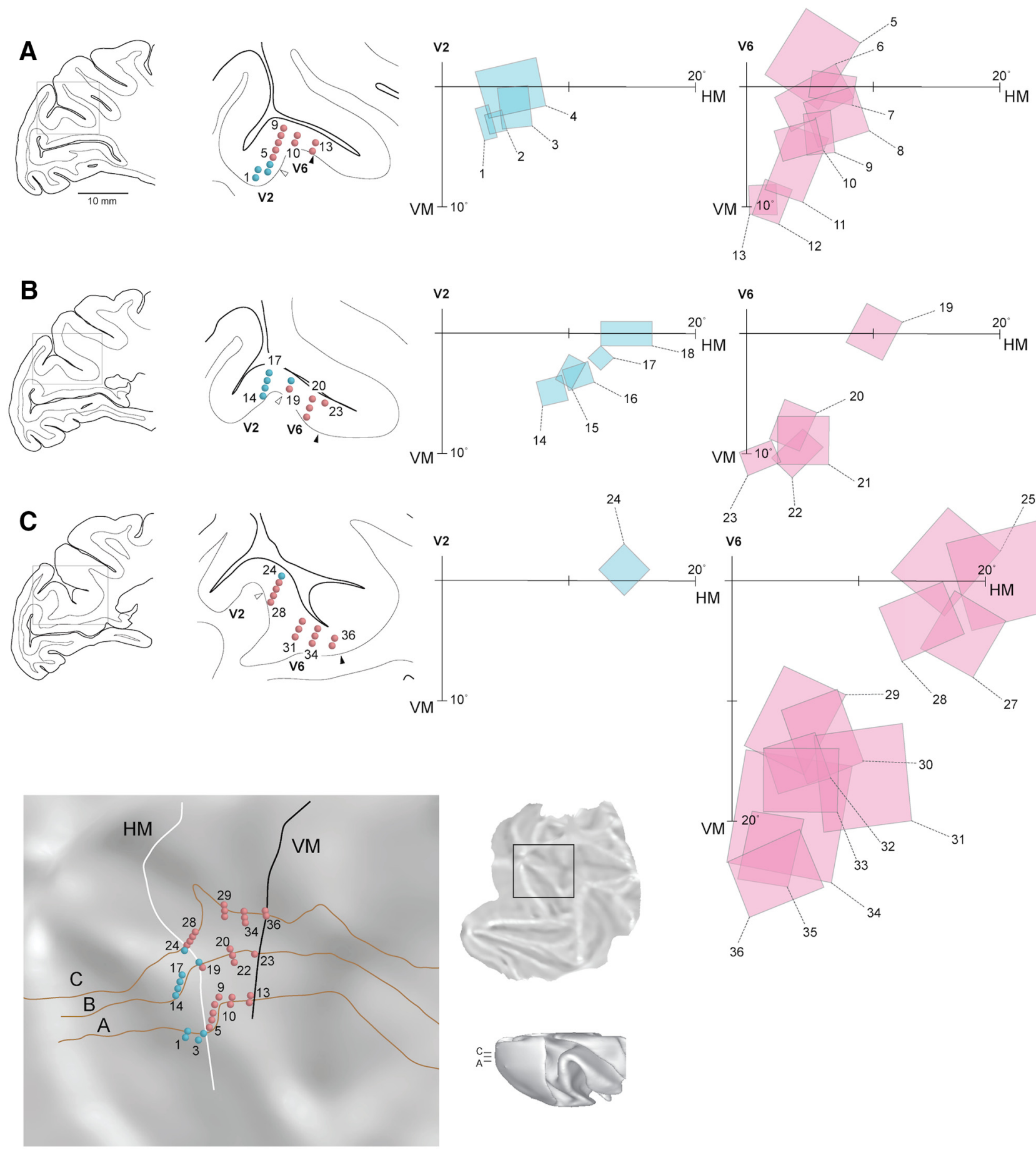

Figure 6. Recording sites and receptive fields obtained from three parasagittal levels $(\boldsymbol{A}-\boldsymbol{C})$ encompassing the annectant gyrus in case 2 . Left, Parasagittal sections with recording sites indicated (blue: recording sites assigned to V2; red: recording sites assigned to V6). Right, Receptive fields recorded in V2 and V6. Bottom left, Flat map illustrating the spatial relationship between recording sites. The cortical midthickness line corresponding to the parasagittal sections corresponding to the three levels is indicated by brown lines, together with the locations of the recording sites. The white and black lines indicate the location of the caudal and rostral borders of $\mathrm{V} 6$, estimated as the locations where the receptive field sequences reverted near the horizontal meridian (HM) and vertical meridian (VM), respectively. The inserts show the location of the reconstructed region in a flat map prepared with CARET and the levels of the three sections in a dorsal view of the brain.

gyrus (a buried gyrus, not visible from the surface of the brain, which separates the lunate sulcus from the posterior ramus of the intraparietal sulcus). The lateral limit of our recording grids included this gyrus.

Figure 5 summarizes the visual topography of the studied region in unfolded two-dimensional reconstructions. The left panels illustrate interpolated polar angle maps, and the right panels show the eccentricity maps. Receptive field sequences that illus- trate in more detail the main points highlighted in the analysis below (i.e., those in V2 and in the redefined V6) are shown in Figures 6, 7, 8, and 9 .

V2

As shown in Figure $5 A-D$, the caudal and rostral borders of dorsal V2 were evident in each animal, coinciding with representations of the vertical (red tones, and continuous lines to the left of each 



Figure 7. Recording sites and receptive fields obtained from two additional parasagittal levels ( $D$ and $E$ ) in case 2 medial to the annectant gyrus. Conventions are as in Figure 6.

map) and horizontal (yellow tones, and dashed lines) meridians. The receptive field eccentricity in the explored region of V2 (Fig. $5 E-H$ ) increased from $\sim 5^{\circ}$ (red) to $60^{\circ}$ or more (dark blue) from lateral to medial. These observations conform well to the findings of earlier studies (Gattass et al., 1981).

\section{Redefined V6, recording sites in the lunate sulcus and annectant gyrus}

Receptive fields obtained in recording sites immediately rostral to $\mathrm{V} 2$ in the lunate sulcus and annectant gyrus were also located in the lower visual field and exhibited a topography that was consistent across animals (Fig. 5A-D). Historically, receptive fields in this region have been attributed to $\mathrm{V} 3 \mathrm{~d}$, and the present data conform with the expected visual topography (Van Essen and Zeki, 1978; Gattass et al., 1988). As progressively more anterior sites were sampled in this region, the receptive fields moved from the vicinity of the horizontal meridian (represented at the V2 border, dashed lines) toward the lower vertical meridian (red tones and right continuous lines in Fig. 5; receptive field sequences A-E in Figs. 6 and 7). Furthermore, consideration of 

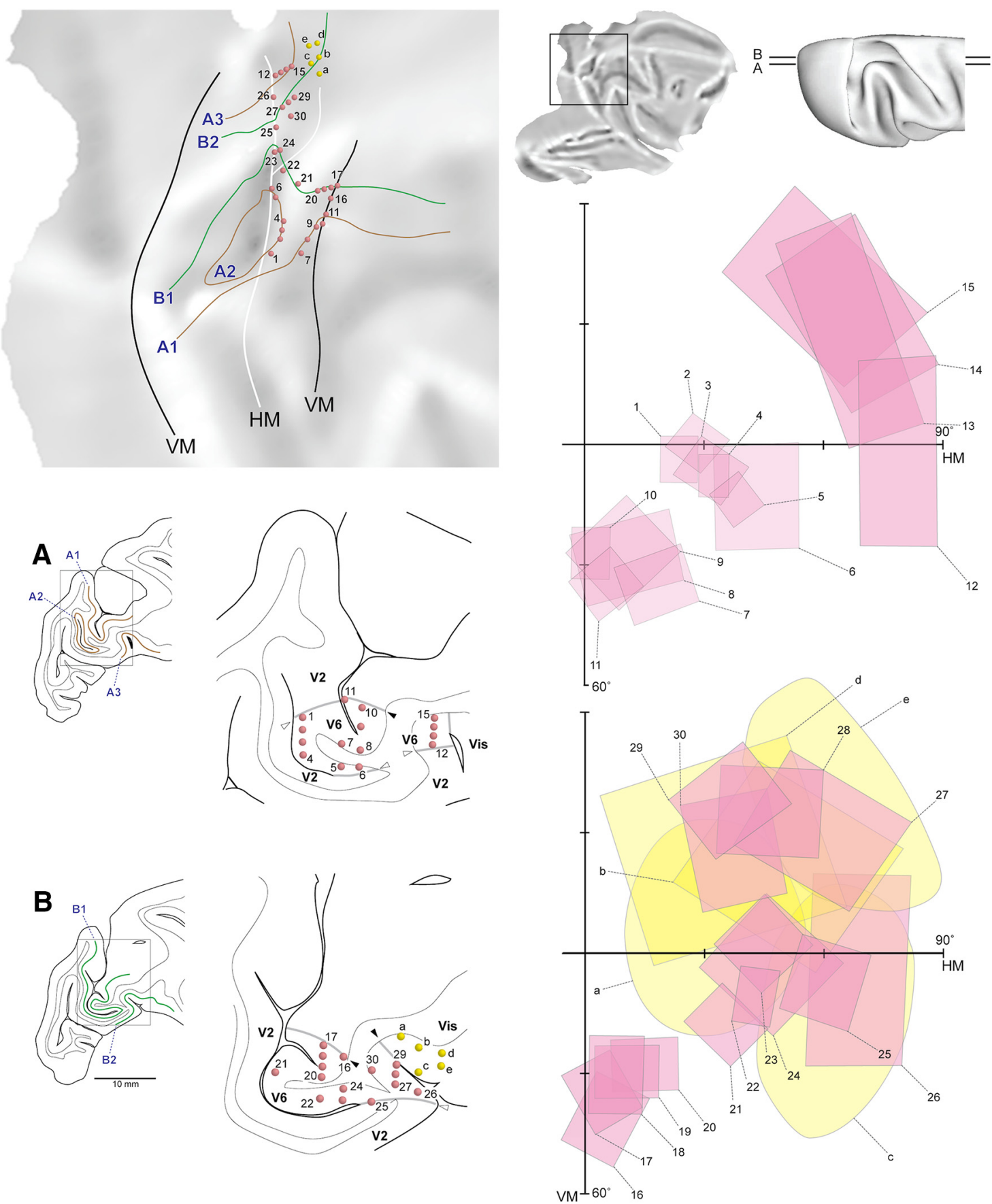

Figure 8. Receptive fields obtained from recording sites near the mesial surface in case 1. Top left, Recording sites shown in an unfolded map of the cortex. The solid and dashed lines indicate representations of the lower vertical meridian (VM) and horizontal meridian (HM), respectively. As indicated in the inserts $(A, B$, left), the complex topology of the cortex in this region meant that recording sites located in different parts of $\mathrm{V} 6$ projected to different midthickness contours ( $\boldsymbol{A} \mathbf{1}-\boldsymbol{A} \mathbf{3}, \boldsymbol{B} 1$ and $\boldsymbol{B 2}$, which are indicated in the flat map using different colors. Bottom left, Location of the same recording sites shown with the same conventions used in Figs. 6 and 7. The red recording sites and receptive fields were assigned to V6 and yellow recording sites to cortex rostral to 66 (Vis). Right, Receptive fields corresponding to the recording sites shown on the left. To facilitate visualization of the far peripheral receptive fields in a planar representation, the receptive fields are represented here as rectangles with area proportional to that obtained using appropriate geodesic calculations (Yu and Rosa, 2010). The inserts on the top right indicate the region magnified in the flat map and the levels of sections $A$ and $B$ in a dorsal view of the brain.

recording sites arranged from lateral (e.g., sequence A in Fig. 6) to medial (e.g., sequence E in Fig. 7) revealed a monotonic increase in receptive field eccentricity without any reversals that would indicate the presence of two distinct lower quadrant representations. The width of the redefined V6 $(3-5 \mathrm{~mm})$ in this region was approximately half that of V2. In three cases, small islands of cortex containing receptive fields centered above the horizontal meridian were observed within the putative limits of the redefined V6 near the lateral limit of the recording grid (light green tones near the bottom of the maps in Fig. $5 A, B, D$ ). However, our recording grid did not include more lateral regions where a more extensive representation of the upper quadrant is reported to exist adjacent to V2 (Zhu and Vanduffel, 2019). Receptive field 5 in Figure $6 \mathrm{~A}$ exemplifies the upper quadrant invasion in this region. 

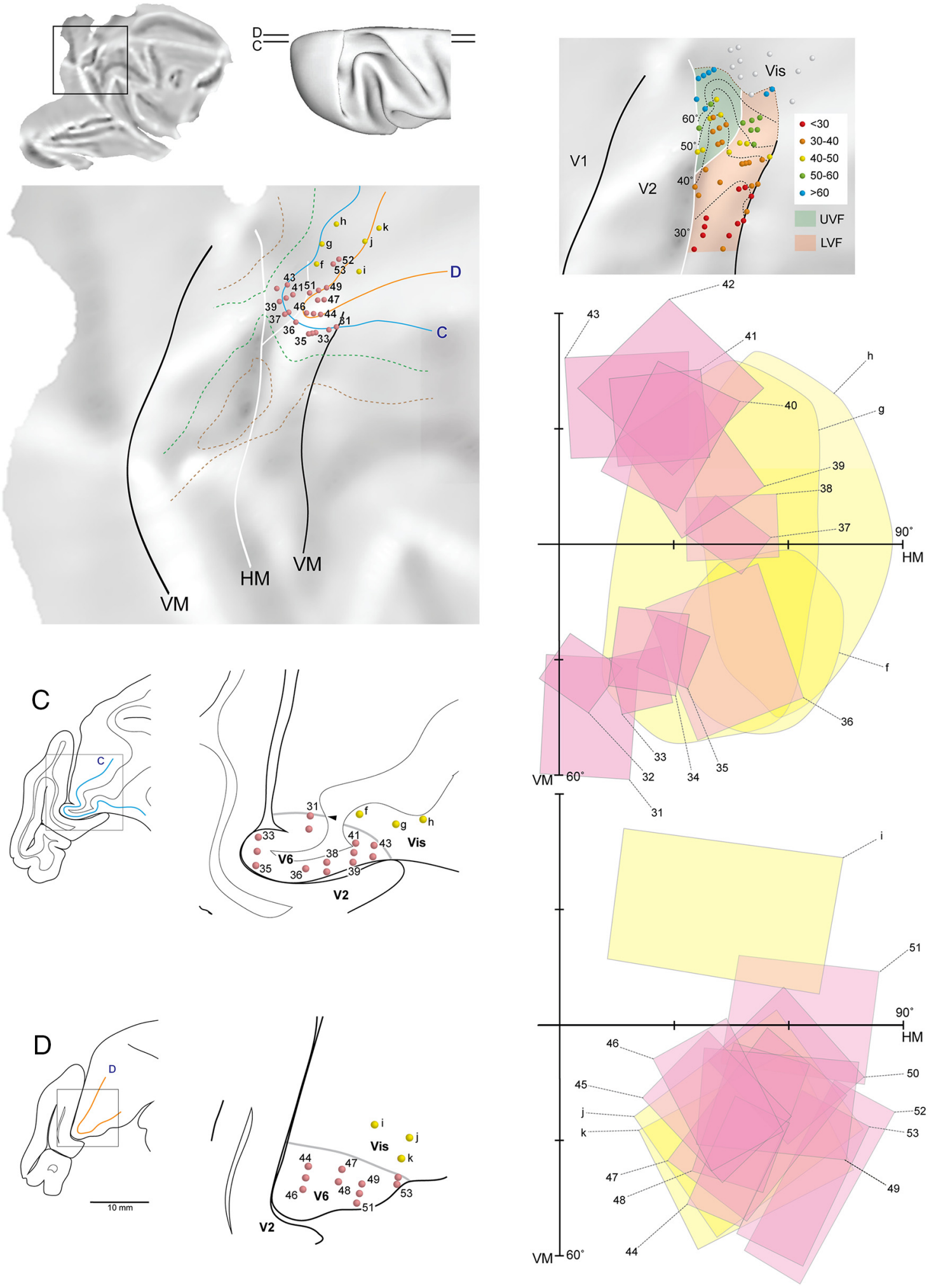

Figure 9. Additional recording sites in V6 obtained in sections ( $C$ and D) medial to those illustrated in Figure 8. Conventions are as in Figure 8. Top right, Summary map showing all recording sites illustrated in Figures 8 and 9 color coded according to eccentricity and location relative to the horizontal meridian. LVF, lower visual field; UVF, upper visual field.

\section{Redefined V6, medial recording sites}

The representation of the horizontal meridian formed the border between V2 and the third-tier cortex until a point near where the rostral bank of the parietooccipital sulcus reaches the midline. Beyond this region (arrowheads in Fig. 5) the representation of the horizontal meridian (dashed lines in Fig. 5) bifurcated, with a posterior branch that followed the rostral border of $\mathrm{V} 2$ and an anterior branch that projected into the mesial surface rostral to the parietooccipital sulcus. The region of cortex between these two branches (including parts of the upper bank of the parietooccipital medial sulcus and of the mesial surface) contained a representation of the periphery $\left(>30^{\circ}\right)$ of the upper visual quadrant 
(green and blue tones in Fig. 5A-D). This upper quadrant representation was found in all four animals, although in case 3 , it seemed to be interrupted by pockets of cells centered in the lower quadrant near the horizontal meridian (Fig. 5C).

The upper quadrant representation region described above has been traditionally assigned to PO/V6, usually being combined with a lower quadrant representation in the parietooccipital sulcus to form a representation of peripheral vision (Colby et al., 1988; Neuenschwander et al., 1994; Gamberini et al., 2015; Fig. 1). However, as illustrated in Figures 5, 8, and 9, our data show that the peripheral lower quadrant map normally assigned to PO/V6 is in fact a straightforward extension of that illustrated in Figures 6 and 7. Namely, receptive fields recorded near the lip of the parietooccipital sulcus (arrowheads in Fig. 5; see also sequence $\mathrm{E}$ in Fig. 7 and sequences $1-5$ in Fig. 8) had eccentricities between $30^{\circ}$ and $40^{\circ}$, whereas cortex located more medially completed the representation up to the temporal edge of the field of vision.

This region of the macaque brain is highly convoluted, making the interpretation of data obtained in single sections complex. The full set of observations from case 1, which yielded the most complete map of this region, are summarized in Figures 8 and 9, which show four parasagittal sections arranged from lateral (A) to medial (D). The key observations are best appreciated in the sequence illustrated in Figure 9, section C. In this section, recording sites obtained in the rostral bank of the parietooccipital sulcus near the rostral border of the densely myelinated zone represent the peripheral part of the vertical meridian at $\sim 40^{\circ}$ eccentricity (e.g., sites 31 and 32). Moving ventral and rostrally toward the dorsal bank of the parietooccipital medial sulcus, the receptive fields move toward the peripheral horizontal meridian (sites 3337 ) and proceed to invade the upper visual field (sites 38-43), forming an arch-like sequence with no evidence of a reversal.

The other panels of Figures 8 and 9 give additional context. In Figure 8, section A illustrates the relationship of V6 to parts of V2 located in the caudal bank of the parietooccipital sulcus, which was cut nearly tangentially in this case. As expected, receptive fields near the V2 border represent the horizontal meridian (e.g., sites 1-6), and progressively more rostral sites move toward the vertical meridian at $\sim 30^{\circ}$ eccentricity ( sites $7-11$ ); this is similar to the sequence illustrated in Figure 7, section $\mathrm{E}$ (obtained in case 2). At a slightly more medial level (Fig. 8, section B), the continuity between lower and upper quadrant representations is already evident (receptive fields and sites 21-30). The location of the far periphery of the upper quadrant encompassing the monocular crescent is evident in section A (sites 12-15). Finally, the remainder of the lower quadrant representation, up to the temporal edge of the monocular crescent, is represented in the dorsal part of the gyrus that separates the rostral bank of the parietooccipital sulcus from the upper bank of the parietooccipital medial sulcus (Fig. 9, section D).

The top right panel in Figure 9 shows a composite of the location of all recording sites illustrated in Figures 8 and 9 encoded by receptive field eccentricity and location relative to the horizontal meridian. In summary, a parsimonious interpretation of the data suggests that the dorsomedial third-tier cortex includes a single complete representation of the lower quadrant, which extends at least from the medial part of the annectant gyrus to the mesial surface. Further, this representation is continuous with a representation of the peripheral upper quadrant. These upper and lower quadrant maps are located in the most densely myelinated region of the dorsomedial cortex (Fig. 2) and form the redefined V6. We have found no representation of the central part of the upper quadrant in the region explored by our recordings, so it is possible that V6 extends further than determined by our data (see discussion below).

In more rostral parts of the dorsal bank of the parietooccipital medial sulcus, the visual responses were more difficult to elicit with the stimuli used in the present study. When visual responses were observed the receptive fields were much larger and their borders were harder to define with precision (receptive fields $\mathrm{a}-\mathrm{k}$ in Figs. 8 and 9). Here, we refer to the region from which these responses were obtained as "Vis" (Kobayashi and Amaral, 2003; Bakola et al., 2017; Passarelli et al., 2018; visual area in the ventral part of the precuneate cortex), as our data were insufficient to define borders of possible subdivisions. This region is likely to overlap with parts of the recently proposed area V2A (Elshout et al., 2018) and with the scene-selective retrosplenial region (Nasr et al., 2011).

\section{Receptive field sizes}

Figure 10 compares the receptive field sizes observed in dorsal V2 (Fig. 10A) and V6 (Fig. 10B). These results confirm that the receptive fields of multiunit clusters in the lower quadrant representation of V6 are on average larger than those in V2 (Gattass et al., 1981, 1988; note that in the latter study, these V6 recording sites were attributed to V3d), but also indicate that the upper quadrant receptive fields recorded in the medial cortex attributed to V6 cannot be distinguished from those in the lower quadrant representation. This result argues against receptive field size being a criterion that could justify the distinction between putative areas $\mathrm{V} 3 \mathrm{~d}$ and $\mathrm{PO} / \mathrm{V} 6$.

The sizes of the peripheral receptive fields that we mapped in the redefined V6 (Fig. 10B) are similar to those reported for neurons V6 by Galletti et al. (1999) despite several methodological differences (e.g., anesthetized vs awake animals, mapping on a hemispheric screen vs tangent screen, and the use of different regression models). For example, at an eccentricity of $32^{\circ}$, the estimates are $15.5^{\circ}$ versus $17.7^{\circ}$ for the present and earlier data, respectively, and at $64^{\circ}$, the corresponding figures are $33.5^{\circ}$ versus $32.3^{\circ}$. However, the near-peripheral receptive fields $(\sim 10-$ $20^{\circ}$ eccentricity) tend to be on average smaller in our samples (despite substantial overlap with the distribution illustrated in Fig. 2 of Galletti et al., 1999). This is likely due to the fact that the earlier study used receptive field size as a criterion to sort putative V3 and V6 recording sites, which is likely to have excluded smaller V6 receptive fields from the calculation. Peripheral receptive fields in V2 (Fig. 10A) were on average larger in our sample than those reported by Galletti et al. (1999), but were comparable to those shown by Gattass et al. (1981), who also used a hemispheric screen to map the receptive fields.

\section{Discussion}

We tested the hypothesis that the cortex rostral to V2 in the macaque dorsomedial cortex contains two subdivisions (V3d and $\mathrm{PO}$, or V6, according to current designations), which form distinct visuotopic maps. This model, which is widely recognized in the current literature, was not supported. Instead, we found that part of the territory usually assigned to V3d, together with $\mathrm{PO} /$ V6, encompasses a single map, which extends to the temporal limit of the field of vision (Fig. 3). The representation of the lower quadrant in this map runs as a strip of cortex parallel to V2 for much of its extent, with a shared border formed by neurons that represent the horizontal meridian. However, as it emerges from the rostral bank of the parietooccipital sulcus into the midline, the lower quadrant representation becomes separated from V2 by 

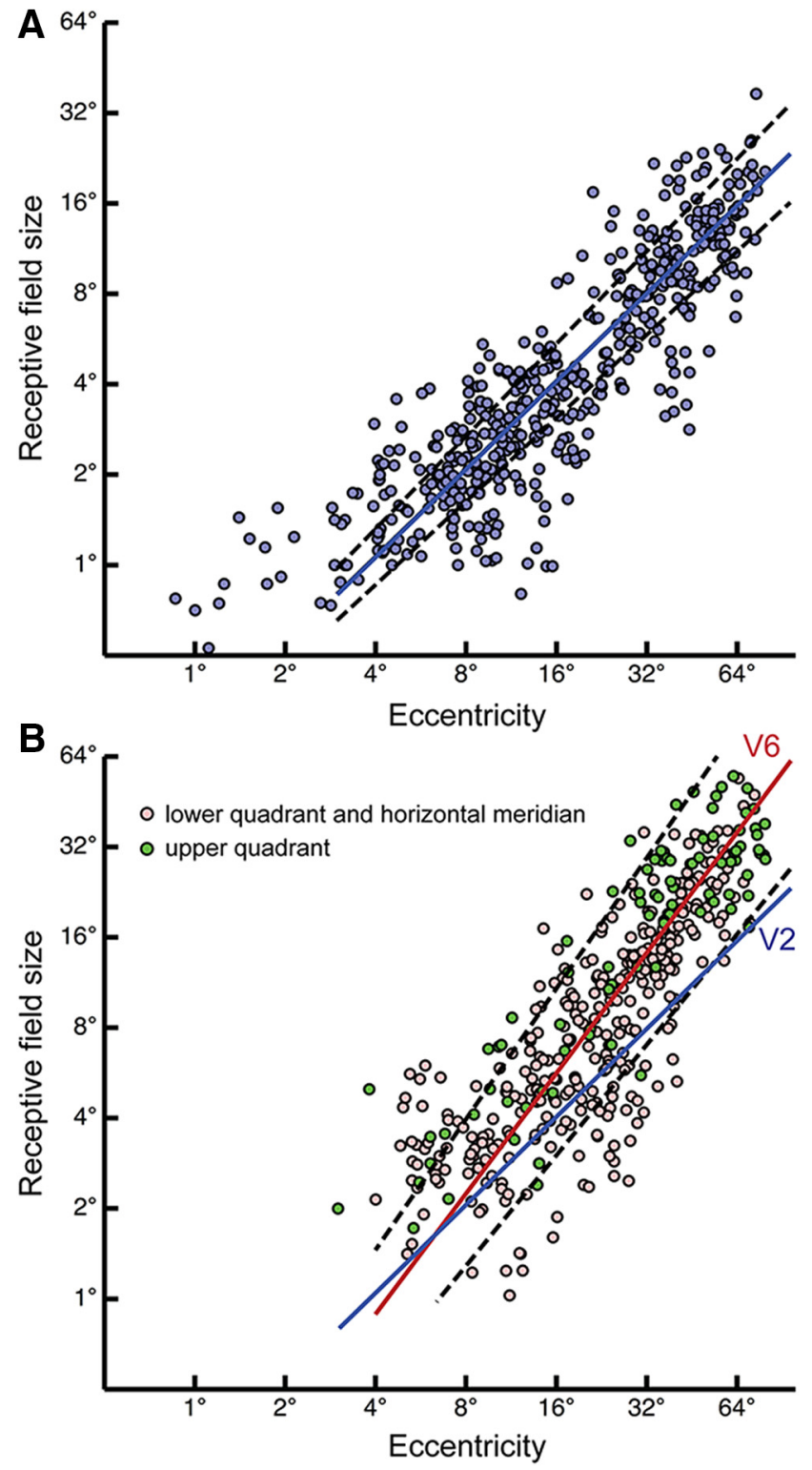

Figure 10. Receptive field size as a function of eccentricity in dorsal V2 (A) and V6 (B). Each graph shows receptive fields from four animals. The best-fitting power functions calculated using a model II (principal axis) regression are illustrated for the lower quadrant representations of V2 (blue line) and V6 (red line). Receptive fields centered on the horizontal meridian were included in this calculation. Confidence intervals (95\%) are indicated by dashed lines. The slopes of the functions calculated for lower quadrant V2 and V6 are clearly different $(p<0.001$, permutation test). The number of receptive fields centered on the upper quadrant was much smaller and nonuniformly distributed across eccentricities, preventing robust statistical comparison. However, the corresponding points (green) overlap extensively with those representing receptive fields in the lower quadrant and horizontal meridian (red).

a region of cortex that represents the periphery of the upper quadrant. This representation of the peripheral upper quadrant forms a continuation of the adjacent lower quadrant representation and is similar to this representation in terms of receptive field size and myeloarchitecture. Therefore, we propose that the region of third-tier cortex of the macaque from the annectant gyrus to the midline is best described by a model that proposes a single area, for which we suggest the name V6 (Fig. 3).

Much of the representation of the lower quadrant uncovered by our data has been traditionally regarded as part of V3 (Burkhalter et al., 1986; Ungerleider and Desimone, 1986; Gat- tass et al., 1988), whereas the medial region where both the upper and the lower quadrant periphery are represented has been assigned to either PO (Colby et al., 1988) or V6 (Galletti et al., 1999). Comparison with published results strongly indicates that the revised area we propose overlaps well with these subdivisions (Fig. 11). However, our results show no reversals or rerepresentation at their putative border. We also found no evidence for an additional area (PIP) inserted between the cortex previously assigned to V3d and PO/V6 (Felleman and Van Essen, 1991; Lewis and Van Essen, 2000.

Our interpretation is supported by well documented anatomical similarities among V3d, V6, and PO, reviewed previously (Rosa et al., 2005, 2009; Angelucci and Rosa, 2015). Each of these putative areas has been described as being heavily myelinated relative to adjacent cortex (see also Fig. 3 ) and to receive a dense projection from layer $4 \mathrm{~b}$ of $\mathrm{V} 1$, which makes them different from ventral and lateral parts of the third-tier complex (Colby et al., 1988; Felleman et al., 1997; Galletti et al., 2001; Jeffs et al., 2015).

\section{Nomenclature and comparison with previous models}

Our findings raise the issue of what is the most appropriate designation for the dorsomedial third-tier area. On balance, this area most closely resembles area V6 as proposed by Galletti et al. (1999, 2001). Like V6, the area encompasses a lower quadrant representation that is mirror symmetrical to that found in $\mathrm{V} 2$, as well as a peripheral upper quadrant representation in the mesial surface and parietooccipital sulcus. Similar to recent depictions of V6 (Gamberini et al., 2015), the lower quadrant representation is more extensive than that of the upper quadrant. Therefore, to avoid multiplication of names, we favor retaining the designation V6 with the provision that, according to the present data, this area extends even more laterally than previously recognized and is not separated from V2 by putative V3 (Gamberini et al., 2015). In comparison, we regard the designation $\mathrm{PO}$ as less satisfactory. As discussed by Galletti et al. (2005), this area, as depicted in the original studies (Colby et al., 1988), encompasses parts of the parietooccipital sulcus that are now known to belong to a functionally different area, V6Av (Passarelli et al., 2011; Fig. 11).

Another viable option would be to retain the name $\mathrm{V} 3 \mathrm{~d}$, which has historical precedence (Ungerleider and Desimone, 1986). However, we believe that this option would increase the potential for confusion given that this designation is most commonly used to refer to the lower quadrant representation of a much more extensive putative $\mathrm{V} 3$, which is proposed to extend all the way to the ventral surface of the brain (Gattass et al., 1988; Lyon and Connolly, 2012). Here, it is important to note that our redefinition of the extent of V6 does not rule out the existence of a V3-like area, albeit smaller than previously proposed. As discussed by Angelucci and Rosa (2015), the macaque homolog of human V3 is likely to be located lateral to the redefined V6 (Fig. 3 ), including parts of the lunate sulcus as well as the lateral and ventral surfaces of the occipital lobe (see also Rosa et al., 2000, 2013, and Jeffs et al., 2015, for evidence in New World monkeys). Adopting the designation V3 to refer to this area is also more satisfactory in terms of homology with V3 in other mammals (also known as area 19; Tusa et al., 1979), which is involved in the analysis of form and most closely aligned with the ventral stream of processing (Tanaka et al., 1987; Dreher et al., 1996; Khayat et al., 2000; Rosa and Manger, 2005). Despite the differences in nomenclature, our results align with the fact that there are marked anatomical and functional differences between the dorsal and ventral components of the macaque third-tier cortex (Burkhalter et al., 1986), with the presently redefined V6 match- 


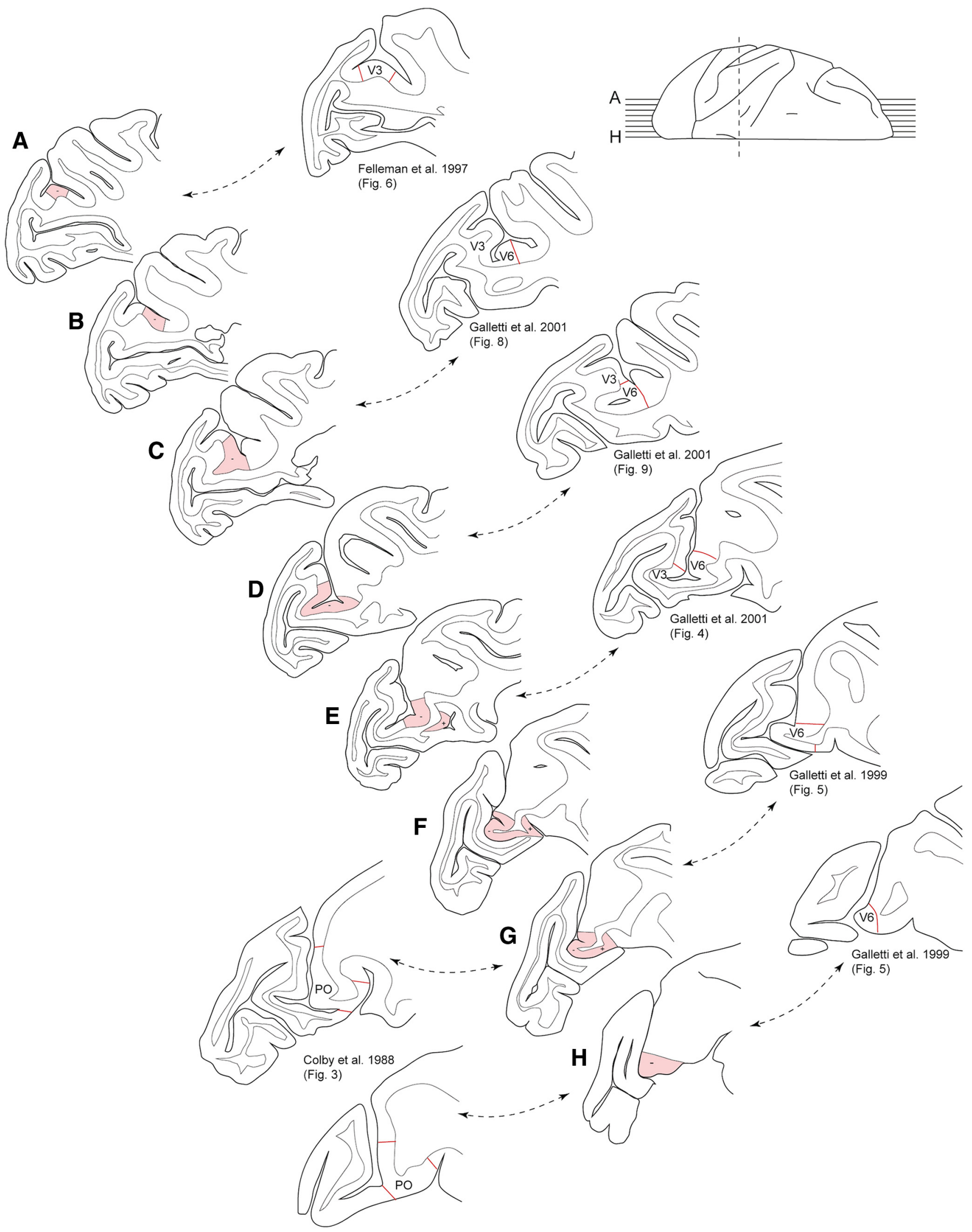

Figure 11. $\mathbf{A}-\boldsymbol{H}$, Parasagittal sections showing the extent of the redefined area $\mathrm{V} 6$ according to the present results (pink). For comparison, the left and right columns show drawings of sections from previous studies (Colby et al., 1988; Felleman and Van Essen, 1997; Galletti et al., 1999, 2001), which show the proposed extents of V3, V6, and P0 (red lines indicating borders of these areas as illustrated in the original studies). 
ing the characteristics of the dorsal component explored by Felleman and Van Essen (1987) and Felleman et al. (1997).

\section{Unresolved issues and limitations}

The present study leaves open the question of the possible location of a representation of the central part of the upper quadrant, which would make the macaque V6 a complete map of the visual field. However, as discussed below, there is evidence from other studies in both New World and Old World monkeys indicating that the "missing" central upper quadrant is likely to be represented more laterally in the lunate sulcus (see also Rosa and Tweedale, 2001; Zhu and Vanduffel, 2019). Although we did observe a few receptive fields centered in the upper quadrant near the lateral end of our explored region, the sample was insufficient to ascertain the extent of this representation.

A second issue that deserves further study is the possibility that there is more than one representation of the upper periphery in the region that we currently assign to V6. Previous studies in New World monkeys have reported a thin strip of transitional upper quadrant representation (area POm) between V2 and area PO (Neuenschwander et al., 1994), and some of our data suggest that a representation of the upper vertical meridian may exist in the corresponding location in the macaque (e.g., receptive field 43 in Fig. 9); this would result in mirror-symmetrical representations of the upper quadrant within the limits of V6. However, given the combination of relatively large receptive fields and scatter, the most parsimonious interpretation of the current data is that a single representation exists. Targeted recordings focused at the V2/V6 border in the parietooccipital medial sulcus would be needed to resolve this issue.

More generally, our study was designed to explore a relatively large area of cortex with densely distributed electrode penetrations in a relatively short time (each experiment encompassing a single acute session $4-5 \mathrm{~d}$ long). This approach allowed accurate reconstruction of the recording sites while still enabling precise delimitation of receptive fields; we regarded these as necessary conditions to test the conflicting models of the organization of the dorsomedial cortex. However, this approach does not always result in uniform coverage given the complex topology of the lunate/parietooccipital/intraparietal cleft, and is intrinsically limited in terms of the volume of cortex that can be covered. Therefore, although the general pattern of representation observed was consistent, the quality of the sampling varied from region to region and from case to case. Whether apparent differences in the detailed maps (e.g., the extent of the upper quadrant representation in medial V6) are real or result from such sampling artifacts will require more study. Considering the complex topology of the region of interest, these studies will preferably involve sectioning the brain in different planes to facilitate the visualization of transitions between areas in different regions. The present data provide a testable hypothesis that can be addressed by functional imaging studies designed for this purpose combining stimulation of the peripheral visual field and high-field MRI.

\section{Comparison with New World monkeys}

Some of the features of the revised V6 resemble those of area DM, as defined in the marmoset monkey (Rosa et al., 2005; Lui et al., 2006). Like the revised macaque V6, DM also shows a continuous complete representation of the lower quadrant adjacent to V2, adjoined by a representation of the peripheral upper quadrant in the midline cortex (Fig. 3; Rosa and Tweedale, 2001). DM also shares the dense myelination of V6 and its specific, dense input from layer $4 \mathrm{~b}$ of V1. Moreover, the connections of DM appear identical to those of $\mathrm{V} 3 \mathrm{~d}$ and $\mathrm{PO} / \mathrm{V} 6$ if one allows for the interpretation that these correspond to the central and peripheral representations of a same area (Rosa et al., 2009). However, as discussed above, our data may not have established the full extent of macaque $\mathrm{V} 6$ given that the electrode penetrations did not extend more laterally into the banks of the lunate sulcus, where a representation of the central upper quadrant is expected to exist (meta-analysis by Angelucci and Rosa, 2015; Zhu and Vanduffel, 2018). Therefore, although the present results indicate that the medial part of V3d and PO/V6 are best considered as parts of the same area, establishing the extent of the similarity of this area to the New World monkey DM will require further work. Earlier studies (Beck and Kaas, 1999) proposed that the designation DM would also be appropriate for this part of the macaque cortex and we suggest that V6 and DM are likely to be designations for homologous areas in the macaque and marmoset.

Although our data were not sufficient to establish a full picture of the organization of areas rostral to the revised V6, they clearly demonstrate that visual responses can be obtained in anesthetized preparations and reveal some similarities with previous studies. For example, in case 1, the midline recording sites rostral to the border of V6 contained visually responsive neurons with very large receptive fields (Figs. 8, 9) that represented both the upper and lower quadrants of the visual field, similar to human area V2A (Elshout et al., 2018).

\section{Conclusions}

Our findings point to a simpler model of the organization of the dorsomedial cortex anterior to area $\mathrm{V} 2$ in macaques, which consolidates subdivisions proposed by different investigators into a single area. We propose retaining the designation V6 for this area while acknowledging the likelihood that it is homologous to area DM in New World monkeys. This model provides a parsimonious account of previous anatomical and electrophysiological observations, including differences between the dorsal and ventral components of the third-tier complex (Burkhalter et al., 1986; Beck and Kaas, 1999; Rosa et al., 2005; Jeffs et al., 2015). Knowing the extent of its application to the human brain will require highfield imaging studies that include stimulation of the far periphery of the visual field, preferably combined with appropriate structural and functional connectivity experiments.

\section{References}

Allman JM, Kaas JH (1975) The dorsomedial cortical visual area: a third tier area in the occipital lobe of the owl monkey (Aotus trivirgatus). Brain Res 100:473-487.

Angelucci A, Rosa MG (2015) Resolving the organization of the third tier visual cortex in primates: a hypothesis-based approach. Vis Neurosci 32: E010.

Bakola S, Passarelli L, Huynh T, Impieri D, Worthy KH, Fattori P, Galletti C, Burman KJ, Rosa MGP (2017) Cortical afferents and myeloarchitecture distinguish the medial intraparietal area (MIP) from neighboring subdivisions of the macaque cortex. eNeuro 4:ENEURO.0344-17.

Beck PD, Kaas JH (1999) Cortical connections of the dorsomedial visual area in Old World macaque monkeys. J Comp Neurol 406:487-502.

Bourne JA, Rosa MGP (2003) Preparation for the in vivo recording of neuronal responses in the visual cortex of anaesthetised marmosets (Callithrix jacchus). Brain Res Brain Res Protoc 11:168-177.

Burkhalter A, Felleman DJ, Newsome WT, Van Essen DC (1986) Anatomical and physiological asymmetries related to visual areas V3 and VP in macaque extrastriate cortex. Vision Res 26:63-80.

Chaplin TA, Yu HH, Soares JG, Gattass R, Rosa MGP (2013a) A conserved pattern of differential expansion of cortical areas in simian primates. J Neurosci 33:15120-15125.

Chaplin TA, Yu HH, Rosa MGP (2013b) Representation of the visual field in the primary visual area of the marmoset monkey: magnification fac- 
tors, point-image size, and proportionality to retinal ganglion cell density. J Comp Neurol 521:1001-1019.

Cignoni P, Corsini M, Ranzuglia G (2008) Meshlab: an open source 3D mesh processing system. ERCIM News. Available at http://meshlab. sourceforge.net/.

Colby CL, Gattass R, Olson CR, Gross CG (1988) Topographical organization of cortical afferents to extrastriate visual area PO in the macaque: a dual tracer study. J Comp Neurol 269:392-413.

Dreher B, Wang C, Burke W (1996) Limits of parallel processing: excitatory convergence of different information channels on single neurons in striate and extrastriate visual cortices. Clin Exp Pharmacol Physiol 23: 913-925.

Elshout JA, van den Berg AV, Haak KV (2018) Human V2A: a map of the peripheral visual hemifield with functional connections to scene-selective cortex. J Vis 18:22.

Felleman DJ, Van Essen DC (1987) Receptive fields properties of neurons in area V3 of macaque monkey extrastriate cortex. J Neurophysiol 57: 889-920.

Felleman DJ, Van Essen DC (1991) Distributed hierarchical processing in the primate cerebral cortex. Cereb Cortex 1:1-47.

Felleman DJ, Burkhalter A, Van Essen DC (1997) Cortical connections of areas V3 and VP of macaque monkey extrastriate visual cortex. J Comp Neurol 379:21-47.

Galletti C, Fattori P, Gamberini M, Kutz DF (1999) The cortical visual area V6: brain location and visual topography. Eur J Neurosci 11:3922-3936.

Galletti C, Gamberini M, Kutz DF, Fattori P, Luppino G, Matelli M (2001) The cortical connections of area V6: an occipito-parietal network processing visual information. Eur J Neurosci 13:1572-1588.

Galletti C, Gamberini M, Kutz DF, Baldinotti I, Fattori P (2005) The relationship between V6 and PO in macaque extrastriate cortex. Eur J Neurosci 21:959-970.

Gallyas F (1979) Silver staining of myelin by means of physical development. Neurol Res 1:203-209.

Gamberini M, Fattori P, Galletti C (2015) The medial parietal occipital areas in the macaque monkey. Vis Neurosci 32:E013.

Gattass R, Gross CG, Sandell JH (1981) Visual topography of V2 in the macaque. J Comp Neurol 201:519-539.

Gattass R, Sousa AP, Gross CG (1988) Visuotopic organization and extent of V3 and V4 of the macaque. J Neurosci 8:1831-1845.

Jeffs J, Federer F, Angelucci A (2015) Corticocortical connection patterns reveal two distinct visual cortical areas bordering dorsal V2 in marmoset monkey. Vis Neurosci 32:E012.

Khayat PS, Saint-Amour D, Molotchnikoff S, Lepore F, Guillemot JP (2000) Cellular response to texture and form defined by motion in area 19 of the cat. Eur J Neurosci 12:1727-1738.

Kobayashi Y, Amaral DG (2003) Macaque monkey retrosplenial cortex: II. Cortical afferents. J Comp Neurol 466:48 -79.

Lewis JW, Van Essen DC (2000) Mapping of architectonic subdivisions in the macaque monkey, with emphasis on parieto- occipital cortex. J Comp Neurol 428:79-111.

Lui LL, Bourne JA, Rosa MGP (2006) Functional response properties of neurons in the dorsomedial visual area of New World monkeys (Callithrix jacchus). Cereb Cortex 16:162-177.

Lyon DC, Connolly JD (2012) The case for primate V3. Proc Biol Sci 279:625-633.

Nasr S, Liu N, Devaney KJ, Yue X, Rajimehr R, Ungerleider LG, Tootell RB (2011) Scene-selective cortical regions in human and nonhuman primates. J Neurosci 31:13771-13785.

Neuenschwander S, Gattass R, Sousa AP, Pinon MC (1994) Identification and visuotopic organization of areas PO and POd in Cebus monkey. J Comp Neurol 340:65-86.

Passarelli L, Rosa MGP, Gamberini M, Bakola S, Burman KJ, Fattori P, Gal- letti C (2011) Cortical connections of area V6Av in the macaque: a visual-input node to the eye/hand coordination system. J Neurosci 31:1790-1801.

Passarelli L, Rosa MGP, Bakola S, Gamberini M, Worthy KH, Fattori P, Galletti C (2018) Uniformity and diversity of cortical projections to precuneate areas in the macaque monkey: what defines area PGm? Cereb Cortex 28:1700-1717.

Pitzalis S, Galletti C, Huang RS, Patria F, Committeri G, Galati G, Fattori P, Sereno MI (2006) Wide-field retinotopy defines human cortical visual area V6. J Neurosci 26:7962-7973.

Pitzalis S, Sereno MI, Committeri G, Fattori P, Galati G, Patria F, Galletti C (2010) Human V6: the medial motion area. Cereb Cortex 20:411-424.

Rosa MGP, Manger PR (2005) Clarifying homologies in the mammalian cerebral cortex: the case of the third visual area (V3). Clin Exp Pharmacol Physiol 32:327-339.

Rosa MGP, Schmid LM (1995) Visual areas in the dorsal and medial extrastriate cortices of the marmoset. J Comp Neurol 359:272-299.

Rosa MGP, Tweedale R (2001) The dorsomedial visual areas in New World and Old World monkeys: homology and function. Eur J Neurosci 13:421-427.

Rosa MGP, Sousa AP, Gattass R (1988) Representation of the visual field in the second visual area in the Cebus monkey. J Comp Neurol 275:326-345.

Rosa MGP, Fritsches KA, Elston GN (1997) The second visual area in the marmoset monkey: visuotopic organisation, magnification factors, architectonical boundaries, and modularity. J Comp Neurol 387:547-567.

Rosa MGP, Piñon MC, Gattass R, Sousa AP (2000) “Third tier” ventral extrastriate cortex in the New World monkey Cebus apella. Exp Brain Res 132:287-305

Rosa MGP, Palmer SM, Gamberini M, Tweedale R, Piñon MC, Bourne JA (2005) Resolving the organization of the New World monkey third visual complex: the dorsal extrastriate cortex of the marmoset (Callithrix jacchus). J Comp Neurol 483:164-191.

Rosa MGP, Palmer SM, Gamberini M, Burman KJ, Yu HH, Reser DH, Bourne JA, Tweedale R, Galletti C (2009) Connections of the dorsomedial visual area: pathways for early integration of dorsal and ventral streams in extrastriate cortex. J Neurosci 29:4548-4563.

Rosa MGP, Angelucci A, Jeffs J, Pettigrew JD (2013) The case for a dorsomedial area in the primate 'third-tier' visual cortex. Proc Biol Sci 280: 20121372.

Sereno MI, McDonald CT, Allman JM (1994) Analysis of retinotopic maps in extrastriate cortex. Cereb Cortex 4:601-620.

Sereno MI, Dale AM, Reppas JB, Kwong KK, Belliveau JW, Brady TJ, Rosen BR, Tootell RB (1995) Borders of multiple visual areas in humans revealed by functional magnetic resonance imaging. Science 268:889-893.

Tanaka K, Ohzawa I, Ramoa AS, Freeman RD (1987) Receptive field properties of cells in area 19 of the cat. Exp Brain Res 65:549-558.

Tusa RJ, Rosenquist AC, Palmer LA (1979) Retinotopic organization of areas 18 and 19 in the cat. J Comp Neurol 185:657-678.

Ungerleider LG, Desimone R (1986) Cortical projections of visual area MT in the macaque. J Comp Neurol 248:190-222.

Van Essen DC, Drury HA, Dickson J, Harwell J, Hanlon D, Anderson CH (2001) An integrated software suite for surface-based analyses of cerebral cortex. J Am Med Inform Assoc 8:443-459.

Van Essen DC, Zeki SM (1978) The topographic organization of rhesus monkey prestriate cortex. J Physiol 277:193-226.

Yu HH, Rosa MGP (2010) A simple method for creating wide-field visual stimulus for electrophysiology: mapping and analyzing receptive fields using a hemispheric display. J Vis 10:15.

Zhu Q, Vanduffel W (2019) Submillimeter fMRI reveals a layout of dorsal visual cortex in macaques, remarkably similar to New World monkeys. Proc Natl Acad Sci U S A 116:2306-2311. 